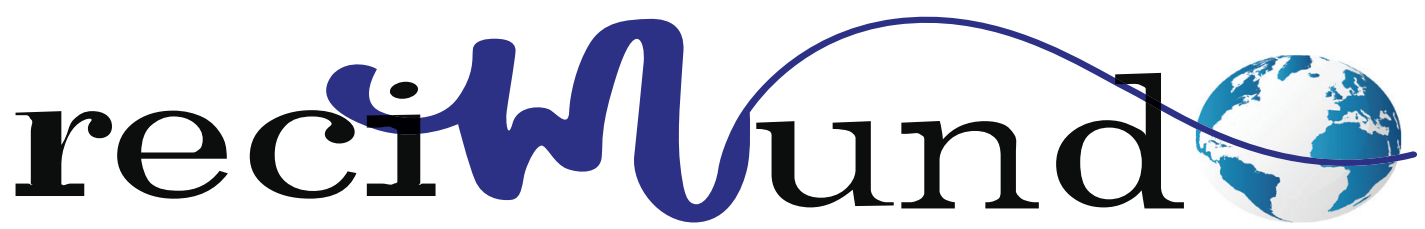

Revista Científica Mundo de la Investigación y el Conocimiento

DOI: 10.26820/recimundo/5.(2).abril.2021.37-45

URL: https://recimundo.com/index.php/es/article/view/1032

EDITORIAL: Saberes del Conocimiento

REVISTA: RECIMUNDO

ISSN: 2588-073X

TIPO DE INVESTIGACIÓN: Artículo de revisión

CÓDIGO UNESCO: 32 Ciencias Médicas

PAGINAS: 37-45

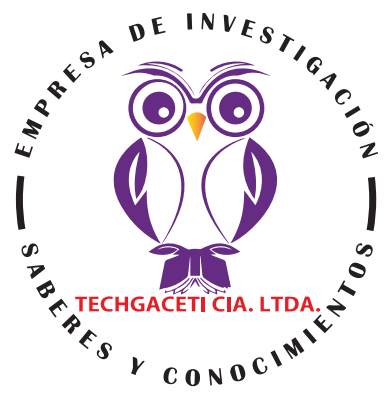

\title{
Oxigenoterapia a alto flujo en COVID-19
}

High-flow oxygen therapy in COVID-19

Oxigenoterapia de alto fluxo na COVID-19

Tangerine Kathina Pozo Rivadeneira'; María Fernanda Matute Solís'; Franklin Iván Moreno Castro3; Jonathan Alberto Castillo Olvera ${ }^{4}$

\section{RECIBIDO: 15/01/2021 ACEPTADO: 20/03/2021 PUBLICADO: 01/04/2021}

1. Médico de la Universidad Católica de Santiago de Guayaquil; Investigador Independiente; Guayaquil, Ecuador; tkpr93@ gmail.com; (D) https://orcid.org/0000-0002-8830-0870

2. Médico de la Universidad Católica de Santiago de Guayaquil; Investigador Independiente; Guayaquil, Ecuador; mafermatute14@gmail.com; (iD https://orcid.org/0000-0002-7488-7797

3. Médico de la Universidad Católica de Santiago de Guayaquil; Investigador Independiente; Guayaquil, Ecuador; frankfimcmoreno@gmail.com; (iD https://orcid.org/0000-0002-4363-2775

4. Médico de la Universidad de Guayaquil; Investigador Independiente; Guayaquil, Ecuador; jonathan_athlerm@hotmail.com; (iD) https://orcid.org/0000-0002-1089-9297

\section{CORRESPONDENCIA}

Tangerine Kathina Pozo Rivadeneira

tkpr93@gmail.com

Guayaquil, Ecuador

(C) RECIMUNDO; Editorial Saberes del Conocimiento, 2021 


\section{RESUMEN}

La situación más crítica de los pacientes infectados por COVID-19, es cuando se desarrolla insuficiencia respiratoria, es aquí donde se generan los mayores índices de mortalidad. Hay diferentes técnicas para la oxigenación de los pacientes, están los procedimientos no invasivos como la cánula nasal de alto flujo y la ventilación mecánica no invasiva, y los procedimientos contrarios como la intubación orotraqueal. Sin embargo, en el mundo medico sigue habiendo posiciones encontradas acerca de oxigenoterapia de alto flujo. La metodología de la investigación, es una revisión bibliográfica apoyada por medios electrónicos, como fuente primaria de la obtención de la información. Las conclusiones mas relevantes es que la oxigenación de alto flujo es mas recomendada en la hipoxia leve, ya que puede reducir los índices de mortalidad por medio de una mejor oxigenación y reducción del trabajo respiratorio, sin embargo, de no tener éxito, hay que proceder a la intubación. La bibliografía consultada infiere que los pacientes jóvenes tienen más altas posibilidades de recuperación por la oxigenación de alto flujo. La oxigenoterapia de alto flujo es riesgosa para el personal médico, ya que se pueden generar aerosoles como consecuencia de la tos del paciente que infieren una alta probabilidad de contagio, sin embargo, esta se puede reducir por el uso de mascarilla.

Palabras clave: Oxigenoterapia, flujo, cánula, hipoxia, respiratorio.

\section{ABSTRACT}

The most critical situation of patients infected by COVID-19 is when respiratory failure develops, it is here where the highest mortality rates are generated. There are different techniques for oxygenation of patients, there are non-invasive procedures such as the high-flow nasal cannula and non-invasive mechanical ventilation, and the opposite procedures such as orotracheal intubation. However, in the medical world there are still conflicting positions about high-flow oxygen therapy. The research methodology is a bibliographic review supported by electronic means, as the primary source for obtaining information. The most relevant conclusions is that high-flow oxygenation is more recommended in mild hypoxia, since it can reduce mortality rates through better oxygenation and reduction of work of breathing, however, if it is not successful, proceed to intubation. The bibliography consulted infers that young patients have a higher chance of recovery due to highflow oxygenation. High-flow oxygen therapy is risky for medical personnel, since aerosols can be generated as a result of the patient's cough that infer a high probability of contagion, however, this can be reduced by the use of a mask.

Keywords: Oxygen therapy, flow, cannula, hypoxia, respiratory.

\section{RESUMO}

A situação mais crítica dos pacientes infectados pela COVID-19 é quando a insuficiência respiratória se desenvolve, é aqui que são geradas as maiores taxas de mortalidade. Há diferentes técnicas de oxigenação dos pacientes, há procedimentos não invasivos como a cânula nasal de alto fluxo e ventilação mecânica não invasiva, e os procedimentos opostos como a intubação orotraqueal. Entretanto, no mundo médico ainda existem posições conflitantes sobre a oxigenoterapia de alto fluxo. A metodologia de pesquisa é uma revisão bibliográfica apoiada por meios eletrônicos, como a principal fonte para a obtenção de informações. A conclusão mais relevante é que a oxigenação de alto fluxo é mais recomendada em hipoxia leve, pois pode reduzir as taxas de mortalidade através de uma melhor oxigenação e redução do trabalho de respiração, porém, se não for bem sucedida, proceder à entubação. A bibliografia consultada infere que pacientes jovens têm maior chance de recuperação devido à oxigenação de alto fluxo. A oxigenoterapia de alto fluxo é arriscada para o pessoal médico, já que os aerossóis podem ser gerados como resultado da tosse do paciente que inferem uma alta probabilidade de contágio, porém, isto pode ser reduzido pelo uso de uma máscara.

Palavras-chave: Oxigenoterapia, fluxo, cânula, hipoxia, respiratória. 


\section{Introducción}

La actual pandemia por SARS-Coronavirus-2 está suponiendo una sobrecarga por todos los sistemas sanitarios. Ello ha supuesto un incremento en la presión asistencial en las Unidades de Cuidados Intensivos (UCI) debido, principalmente, al desarrollo de insuficiencia respiratoria aguda (IRA) hipoxémica que precisa soporte ventilatorio. La técnica de soporte ventilatorio más usada es la ventilación mecánica invasiva (88\%), frente a un $11 \%$ de la ventilación mecánica no invasiva (VNI). Con base en epidemias previas (SARS, influenza A-H1N1) (Belenguer-Muncharaz \& Hernández-Garcés, 2020).

La prevalencia de insuficiencia respiratoria hipóxica (IRAH) en pacientes con COVID-19 es del 19\%, con informes recientes de China que mostraron que del $4 \%$ al $13 \%$ de los pacientes con COVID-19 recibieron ventilación con presión positiva no invasiva (VMNI) y del $2,3 \%$ al $12 \%$ requirieron ventilación mecánica invasiva. Aunque la verdadera incidencia de IRAH en pacientes con COVID-19 no está clara, se puede establecer que cerca de un 14\% desarrollará una enfermedad grave que requiera oxigenoterapia, y el 5\% requerirá manejo en la $\mathrm{UCI}$ y ventilación mecánica (Laverde \& Respiratoria, s.f).

Los pacientes con síndrome de dificultad respiratoria aguda secundario a COVID-19 tienen una forma de presentación atípica, con discrepancia entre una mecánica pulmonar aceptable y una hipoxia marcada; pudiéndose explicar por una notable hiperperfusión de tejido pulmonar no ventilado. La respuesta ante la posición prono o el PEEP alto no se debe únicamente al reclutamiento pulmonar, sino al hecho de que estos pacientes -que cursan con una neumonía poco reclutable presentan una redistribución de la perfusión en respuesta a la presión o fuerzas gravitacionales (Mejía-Zuluaga, Duque-González, Orrego-Garay, Escobar-Franco, \& Duque-Ramírez, 2020).
La oxigenoterapia se define como el aporte artificial de oxígeno(O2) en el aire inspirado; su objetivo principal es la mejoría en la oxigenación tisular, que se consigue cuando la presión parcial de $\mathrm{O} 2$ (PO2) en la sangre arterial supera los $60 \mathrm{mmHg}$, lo que se corresponde, aproximadamente, con una saturación de hemoglobina del 90\% (Avendaño \& Healthcare, s.f).

Tabla 1. Consideraciones iniciales respecto a la oxigenación

\begin{tabular}{|c|c|}
\hline Ítem & Observaciones \\
\hline $\begin{array}{c}\text { Consideraciones } \\
\text { iniciales }\end{array}$ & $\begin{array}{l}\text { - } \quad \text { Meta de SaO2 entre } 90 \text { - } 96 \% \\
\text { No tolerar SaO2 < } 90 \text { \% (tienen mal } \\
\text { pronóstico y mayor mortalidad), solo } \\
\text { considerar en caso de EPOC con hi- } \\
\text { poxemia crónica severa asociada. } \\
\text { - } \quad \text { La hiperoxia es igualmente deletérea. } \\
\text { - Se debe buscar normalizar el trabajo } \\
\text { respiratorio del paciente. } \\
\text { Evitar, en lo posible, dispositivos ge- } \\
\text { neradores de aerosoles en sitios sin } \\
\text { aislamiento adecuado. } \\
\text { Evitar nebulizaciones, en caso de } \\
\text { broncoespasmo, usar inhaladores de } \\
\text { dosis medida. } \\
\text { No retrasar la intubación orotraqueal } \\
\text { y la ventilación mecánica en caso de } \\
\text { tener la indicación y disponibilidad } \\
\text { del ventilador (hay que recordar que } \\
\text { son criterios clínicos y gasométricos, } \\
\text { teniendo mayor relevancia o peso, és- } \\
\text { tos últimos). }\end{array}$ \\
\hline $\begin{array}{l}\text { Saturación de } \\
\text { oxígeno }\end{array}$ & $\begin{array}{l}\text { - Requiere de una señal pulsátil. Puede } \\
\text { ser difícil de lograr si hay pobre per- } \\
\text { fusión. } \\
\text { Medición con oxímetro digital u oxí- } \\
\text { metro con sensor en el área frontal de } \\
\text { la cara. } \\
\text { - Si se usa oxímetro digital se deben } \\
\text { despintar las uñas de los pacientes, } \\
\text { para una adecuada medición. } \\
\text { - Meta } \geq 90 \% \text {. } \\
\text { A partir de ésta se puede encontrar } \\
\text { un equivalente de la PaO/FiO }{ }^{2}-> \\
\text { SO2/FiO } \\
\text { - Se calcula a partir de la relación } \\
\quad \text { entre la saturación periférica de } \\
\quad O^{2} \text { y la FiO }{ }^{2} \\
\text { - Meta } \geq 300 \\
\quad \text { Alerta, si } \leq 200 \text {. }\end{array}$ \\
\hline Cardioscopio & $\begin{array}{l}\text { - Se usa tanto en enfermedad cardiaca } \\
\text { como no cardiaca, para: } \\
\text { - Detección de trastornos del rit- } \\
\text { - } \text { Do. } \\
\text { - Identificar prolongación del QT. } \\
\text { - Meta: ritmo sinusal con frecuen- } \\
\text { cia cardíaca entre } 50 \text { y } 110 \mathrm{lpm}\end{array}$ \\
\hline
\end{tabular}




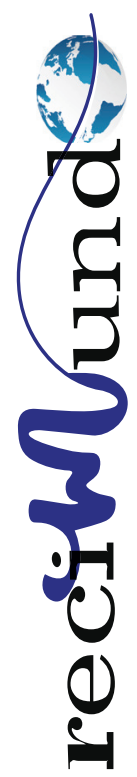

\begin{tabular}{|c|c|}
\hline $\begin{array}{c}\text { Presión arterial no } \\
\text { invasiva }\end{array}$ & $\begin{array}{l}\text { - Verificar que el manguito sea acorde } \\
\text { con el tamaño del paciente. } \\
\text { Siempre tomarla manualmente si hay } \\
\text { cambios súbitos en los valores o si no } \\
\text { detecta la presión del paciente. } \\
\text { PAM = [(PAD } \times 2)+ \text { PAS }] \text { / } 3 \text { o PAM }= \\
1 / 3(\text { PAS - PAD })+\text { PADo Meta PAM } \geq \\
65 \mathrm{~mm} / \mathrm{Hg}\end{array}$ \\
\hline $\begin{array}{l}\text { Frecuencia } \\
\text { respiratoria (FR) }\end{array}$ & $\begin{array}{l}\text { - Calculado a partir de un sensor adhe- } \\
\text { sivo respiratorio o conteo manual de } \\
\text { respiraciones en un minuto. } \\
\text { - Meta }<24 \text { respiraciones por mi- } \\
\text { nuto. }\end{array}$ \\
\hline $\begin{array}{l}\text { Signos de distrés } \\
\text { respiratorio }\end{array}$ & $\begin{array}{l}\text { - } \quad \text { Uso de músculos accesorios. } \\
\text { - } \quad \text { Fatiga. } \\
\text { - } \quad \text { Retreo nasal. } \\
\text { - } \quad \text { Dificultad para completar frases. }\end{array}$ \\
\hline $\begin{array}{c}\text { Examen físico } \\
\text { para la detección } \\
\text { de hipoperfusión }\end{array}$ & $\begin{array}{l}\text { - Gasto urinario: calculado a partir de la } \\
\text { orina producida por el paciente en un } \\
\text { día (por micción espontánea cuantifi- } \\
\text { cada, peso de pañales usados o por } \\
\text { cuantificación de la sonda vesical). } \\
\text { - Meta: } \\
\text { - } \quad-\geq 0,5 \mathrm{~mL} / \mathrm{kg} / \mathrm{h} \text { (mL de orina/ } \\
\quad \text { peso en } \mathrm{kg} \text { del paciente/24 ho- } \\
\quad \text { ras). } \\
\text { - } \quad->500 \mathrm{cc} \text { en } 24 \text { horas. } \\
\text { - Mexamen de piel: } \\
\text { - Ausencia de piel moteada o cia- } \\
\text { - } \quad \text { nusis. } \\
\text { - Exos periféricos palpables. } \\
\text { - Menado capilaro } \\
\text { Meta: <2-3 segundos en menores de } \\
65 \text { años y menos de } 4,5 \text { segundos en } \\
\text { mayores de } 65 \text { años. }\end{array}$ \\
\hline $\begin{array}{c}\text { Saturación } \\
\text { venosa mixta de } \\
\text { oxígeno (SVO2) }\end{array}$ & $\begin{array}{l}\text { - Se toma a partir de una muestra ve- } \\
\text { nosa. } \\
\text { - } \quad \text { Da estimación de la SaO2 que regre- } \\
\text { sa al corazón derecho. } \\
\text { Se correlaciona con la extracción de } \\
\text { O2 y el balance entre entrega (DO2) y } \\
\text { consumo (VO2). } \\
\text { Si la extracción de O2 aumenta, la } \\
\text { SvO2 disminuye si el sistema CV no } \\
\text { compensa las necesidades de aporte } \\
\text { de O2o Meta } 265 \% \text {. }\end{array}$ \\
\hline Gases arteriales & $\begin{array}{l}\text { - Se deben tomar en: } \\
\text { - Pacientes con posibilidad de ventila- } \\
\text { ción invasiva y uno de los siguientes: } \\
\text { - Hipoxemia severa (SaO2/FiO2 } \\
\text { - } \quad \text { Rieosgo y de alteraciones metabó- } \\
\text { licas. } \\
\text { - Estado de poca perfusión ha- } \\
\text { - Ciendo la SaO2 difícil de tomar. } \\
\text { - Condiciones deteriorantes. }\end{array}$ \\
\hline
\end{tabular}

Fuente: (Mejía-Zuluaga, Duque-González, Orrego-Garay, Escobar-Franco, \& Duque-Ramírez, 2020). Elaboración Propia.

\section{Presentación de la neumonía por CO- VID-19}

No hay hallazgos específicos, las imágenes responden a una neumonía viral típica. Se manifiesta comúnmente como opacidades en vidrio esmerilado, distribuidas bilateralmente en bases y periferias. Los hallazgos evolucionan rápidamente, de unilaterales a bilaterales, y la afectación pulmonar se asocia con la gravedad. Los hallazgos pueden estar presentes en individuos asintomáticos o individuos presintomáticos (Colaianni-Alfonso \& Castro-Sayat, 2020). 


\begin{tabular}{|ll|}
\hline \multicolumn{1}{|c|}{ Fenotipo L (Low o bajo) } & \multicolumn{1}{c|}{ Fenotipo $\mathbf{H}$ (High o alto) } \\
\hline Presentación típica de neumonía viral & Presentación posterior a una enfermedad \\
\hline Hipoxemia sin hipercapnia (IRA T1) & Hipoxemia con \pm eliminación de $\mathrm{CO}_{2}$ (IRA T1 y T2) \\
\hline Baja elastancia del sistema respiratorio & Alta elastancia del sistema respiratorio \\
\hline Relación V/Q baja & Relación V/Q alta \\
\hline Bajo potencial de reclutamiento & Alto potencial de reclutamiento \\
\hline Bajo peso pulmonar & Alto peso pulmonar \\
\hline $\begin{array}{l}\text { Puede responder al soporte respiratorio no invasivo (SRN) de } \\
\text { manera precoz }\end{array}$ & No responde al SRNI \\
\hline
\end{tabular}

Figura 1. Fenotipos en COVID-19.

Fuente: (Colaianni-Alfonso \& Castro-Sayat, 2020).

\section{Radiografía de tórax}

- Sombreado bilateral (72,9\%) - mayormente opacidad de vidrio esmerilado $(68,5 \%)$.

- Enfermedad unilateral (25\%).

- Sombreado local irregular.

- Anormalidades intersticiales (hallazgo menos común, $<5 \%$ en algunos estudios).

- Los derrames pleurales son poco frecuentes (Colaianni-Alfonso \& Castro-Sayat, 2020).

\section{Tomografía computarizada de tórax}

- Opacidades de vidrio esmerilado (98\%).

- Patrón reticular.

- Consolidación (patrón crazy paving) (Colaianni-Alfonso \& Castro-Sayat, 2020).

\section{Ultrasonido pulmonar}

Su rol todavía se está definiendo, pero aún no se observan resultados específicos de COVID-19 en los informes de casos. Los casos exhiben presencia de neumonía viral con características que incluyen:

- Línea pleural irregular.

- Líneas B (pueden ser irregulares e incluso confluentes).

- Patrón irregular con preservación bilateral.
- Áreas de pulmón blanco.

- Consolidaciones subpleurales (Colaianni-Alfonso \& Castro-Sayat, 2020).

\section{Metodología}

La metodología de la investigación, es una revisión bibliográfica apoyada en medios electrónicos como paginas web, en donde se encontró una amplia variedad de artículos de investigación que abordan la temática estudiada, el objetivo es recopilar la información mas relevante de dichos autores para realizar una síntesis bien completa, que pueda ayudar a futuras investigaciones.

\section{Resultados}

\section{Terapia nasal de alto flujo (TNAF) en la neumonía por SARS-CoV-2}

La primera experiencia reportada con TNAF la describieron Wang y cols. Incluyeron 17 sujetos a los cuales aplicaron TNAF como tratamiento de primera línea. De los 17 sujetos con TNAF, 7 (41\%) fallaron al tratamiento. Se consideró fallo a la necesidad de escalar el tratamiento a Ventilación mecánica no invasiva (VMNI), donde finalmente 2 pacientes requirieron IOT. La relación P/F en el grupo éxito fue de 209 (179-376) vs 142 (130-188) grupo fracaso $(p=0,03)$ (Colaianni-Alfonso \& Castro-Sayat, 2020). 
Guenancia y cols., mostró su experiencia en Francia con 17 pacientes que recibieron TNAF como tratamiento de primera línea, y la utilización de CPAP en caso de no encontrar mejoría en la SpO2; el 76,46\% respondió al tratamiento y pudieron ser dados de alta con un promedio de 7 días. En Italia también se registró la experiencia del uso de TNAF en pacientes con COVID-19; el estudio demostró un $67,85 \%$ de éxito, si bien la población es pequeña $(n=28)$ los resultados fueron alentadores. Además, no se registraron contagios en el equipo de salud. El estudio de mayor envergadura fue el de Patel y cols., en EE.UU. En él se presenta un estudio retrospectivo sobre el rol de TNAF en pacientes con IRAh moderada-grave por COVID-19. El objetivo primario de este estudio fue evitar la ventilación mecánica invasiva (VMI); los objetivos secundarios fueron medir mortalidad y mejoría de la relación $\mathrm{SaO} 2 / \mathrm{FiO} 2$. Los resultados arrojaron que 104 pacientes $(23,3 \%)$ de 445 ingresados requirieron TNAF. Cuarenta y cinco pacientes requirieron escalonamiento a VMNi o conversión a VMi. Once de los pacientes que requirieron conversión a VMi lograron ser extubados y recibieron TNAF como prevención de fallo y ninguno requirió IOT. Seis de los ocho restantes siguieron tratamiento combinado con VMNi, realizando periodos de descanso con TNAF sin requerimiento de VMi (Colaianni-Alfonso \& Castro-Sayat, 2020).

El uso de cánula nasal de alto flujo (CNAF) y de ventilación mecánica no invasiva (VMNI) ha sido discutido desde antes de la pandemia por su efecto temporal en la hipoxemia, reducción del trabajo respiratorio, bajo impacto en mortalidad, alto riesgo de dispersión viral por la mayor generación de aerosoles y la alta incidencia de intubación posterior a la terapia. Diferentes reportes y estudios durante la pandemia apoyan su uso bajo el contexto de la bioseguridad y el entorno adecuado con presión negativa. En adultos COVID-19 e insuficiencia respiratoria hipoxémica se ha preferido el uso de la CNAF sobre el uso de la VMNI siendo la CNAF más cómoda, mejor tolerada, optimiza la atención del usuario por requerir de menos ajustes y monitoreo que la VMNI más aún si iniciamos el manejo en casos de enfermedad más leve previniendo su deterioro y posible requerimiento de ventilación invasiva, pero sin reducir significativamente la mortalidad y estancia en UCI (Díaz, Scarpetta, Arciniegas, \& Díaz, 2020).

Antes del inicio de la terapia con CNAF o VMNI se debe establecer un paralelo entre las ventajas y los riesgos del uso en COVID -19, prever una posible complicación y tener planeado y listo el equipo para proceder a la intubación orotraqueal (IOT). Durante la terapia se debe tener un estrecho control y monitoreo para identificar si no hay mejoría o si presenta signos o síntomas de deterioro para no continuar el manejo y no retrasar la IOT. Concomitante a una adecuada monitoria y juicio clínico a corto plazo (1 o 2 horas) el uso del índice de ROX (ROX: (SpO2/FIO2) /FR) apoya la evaluación de respuesta a la intervención, con valores es predictor de falla terapéutica e IOT (Díaz, Scarpetta, Arciniegas, \& Díaz, 2020).

En las experiencias reportadas, particularmente en Wuhan, el uso de CNAF se destinó a dos escenarios, el primero de ellos al manejo de la falla moderada, como alternativa para evitar el escalonamiento terapéutico, y el segundo escenario como método de preoxigenación en el momento de la intubación, evitando así el uso de las bolsas auto inflables de presión positiva, por su aumentado riesgo de contaminación al personal de salud. El tratamiento con HFNC se puede seleccionar cuando el dispositivo de oxigenoterapia convencional no puede corregir la hipoxemia y el paciente cumple las siguientes condiciones:

- Hipoxemia leve a moderada $(100 \mathrm{mmHg}$ $\leq \mathrm{PaO} 2$ / FiO $2<300 \mathrm{mmHg}$ ).

- No hay indicaciones para intubación traqueal de emergencia, signos vitales 
relativamente estables; inestabilidad hemodinámica, falla orgánica múltiple o inconsciencia, etc.

- Evite usar HFNC; para pacientes con disfunción leve de ventilación ( $\mathrm{pH}<7.3$ ).

- Los pacientes pueden usarse con precaución, pero deben estar preparados para cambiar a ventilación con presión positiva no invasiva o invasiva en cualquier momento (Laverde \& Respiratoria, s.f).

Para adultos con COVID-19 e insuficiencia respiratoria hipoxémica aguda, sugerimos
el uso de CNAF sobre la oxigenoterapia convencional
En adultos con COVID-19 e insuficiencia respiratoria hipoxémica aguda, sugerimos
usar CNAF sobre VMNI.
En adultos con COVID-19 e insuficiencia respiratoria hipoxémica aguda, si la CNAF
no está disponible y no hay indicación urgente de intubación endotraqueal, se
sugiere un ensayo de VMNI con monitorización estrecha y evaluación a intervalos
cortos para el empeoramiento de la insuficiencia respiratoria.
En adultos con COVID-19 que reciben VMNI o CNAF, se recomienda un estrecho
control para el empeoramiento del estado respiratorio y la intubación temprana en
un entorno controlado, si se produce un empeoramiento.

Figura 2. Recomendaciones de la Society Critical Care Medicine acerca del uso de la CNAF

Fuente: (Laverde \& Respiratoria, s.f).

El estudio publicado por Frat y Col. mostró que la CNAF resultó en una reducción de la mortalidad a los 90 días (OR, 0,42; IC del 95\%, 0,21 a 0,85), aunque en el análisis global de la población no redujo el riesgo de intubación comparado con la oxigenoterapia convencional en pacientes con insuficiencia respiratoria hipóxica aguda. Sin embargo, en el análisis segregado, el grupo de pacientes con una $\mathrm{PaO} 2$ /FIO2 inferior a $200 \mathrm{mmHg}$ si mostro una reducción significativa en la intubación (Laverde \& Respiratoria, s.f).

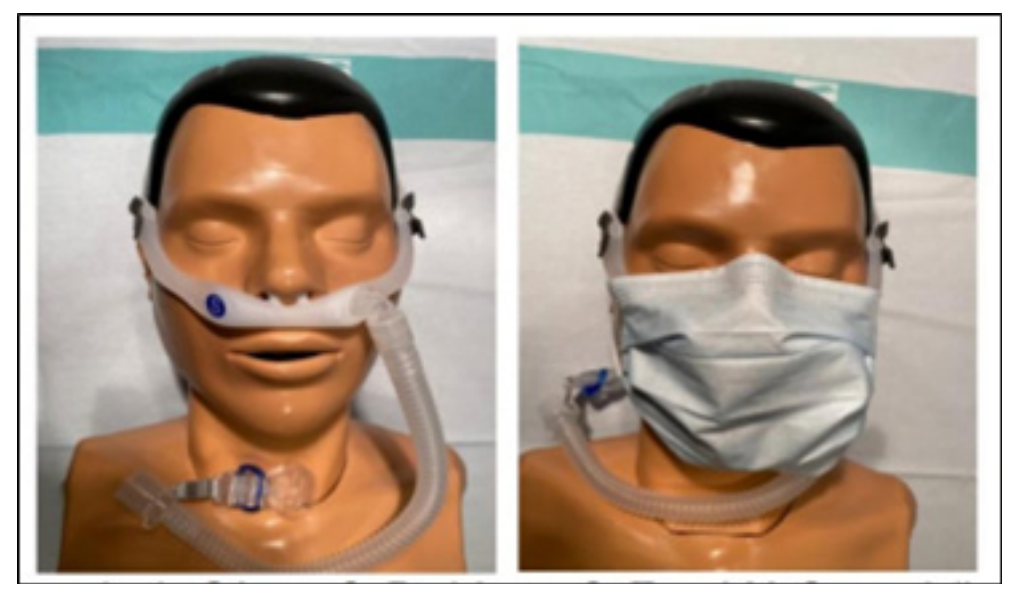

Figura 3. Cánula nasal de alto flujo con mascarilla quirúrgica para evitar emisión de aerosoles (práctica con poca evidencia)

Fuente: (Mosquera, 2020) 
La CNAF es una terapia recomendada para la hipoxia asociada con la enfermedad COVID-19, siempre y cuando el personal lleve los elementos de protección personal (EPP) óptimos junto con las otras precauciones para el control de infecciones nosocomiales. Las salas de presión negativa son preferibles (mas no exclusivas) para los pacientes que reciben terapia con CNAF. Los pacientes con empeoramiento de la hipercapnia, acidemia, fatiga respiratoria, inestabilidad hemodinámica o aquellos con estado mental alterado deben considerarse para la ventilación mecánica invasiva temprana, si corresponde (Laverde \& Respiratoria, s.f).

\section{Conclusión}

En pacientes con COVID-19 se debe evaluar el estado de oxigenación, por medio de la saturación de oxígeno, así como también la clínica del paciente con la frecuencia respiratoria y la presencia de signos de dificultad respiratoria. En base en esto es que se define el tratamiento con oxígeno suplementario.

La oxigenoterapia a alto flujo, es utilizada en pacientes con COVID-19 que hayan desarrollado insuficiencia respiratoria hipoxémica leve y hay mayor compromiso de la $\mathrm{PaO} 2 / \mathrm{FiO} 2$, y esta, dentro de los procedimientos de ventilación no invasivos, sin embargo, este procedimiento de no surtir efecto positivo para el paciente, se pasa a la etapa de la ventilación mecánica asistida, por medio de la intubación orotraqueal.

Sin embargo, en el mundo médico, sigue habiendo discusión acerca del uso de esta técnica, ya que en muchos casos no previene la intubación. Sin embargo, estudios señalan que hay reducción de los índices de mortalidad, ya que esta mejora la oxigenación con la reducción del trabajo respiratorio.
Hay que acotar que, para la realización de este procedimiento, se deben cumplir con altos estándares de bioseguridad, ya que el riesgo de contagio es alto por la generación de aerosoles por la tos del paciente, es por ello que se recomienda que esta terapia sea realizada en habitaciones a puertas cerradas y con ventilación. Sin embargo, se puede recomendar el uso de mascarillas quirúrgicas para reducir las posibilidades de contagio.

\section{Bibliografía}

Avendaño, C., \& Healthcare, G. (s.f). Oxigenoterapia en pacientes adultos positivos para COVID-19.

Belenguer-Muncharaz, A., \& Hernández-Garcés, H. (2020). Fracaso de la ventilación no invasiva tras empleo de oxigenoterapia de alto flujo en pacientes con neumonía por SARS-CoV-2 Failure of non-invasive ventilation after use of high-flow oxygen therapy in patients with SARS-Coronavirus-2 pneumonia. Medicina Invasiva. doi:https://doi.org/10.1016/j.medin.2020.06.012

Colaianni-Alfonso, N., \& Castro-Sayat, M. (2020). Terapia nasal de alto flujo en la insuficiencia respiratoria aguda hipoxémica por SARS-CoV-2. REVISTA DE PATOLOGÍA RESPIRATORIA, 23(3).

Díaz, A. B., Scarpetta, M., Arciniegas, M., \& Díaz, J. (2020). Abordaje del paciente adulto crítico con covid-19. Movimiento Científico, 14(1).

Laverde, J. C., \& Respiratoria, T. (s.f). Soporte respiratorio de alto flujo en el manejo del paciente con COVID-19.

Mejía-Zuluaga, M., Duque-González, L., Orrego-Garay, M., Escobar-Franco, A., \& Duque-Ramírez, M. (2020). Oxigenoterapia en COVID-19: herramientas de uso previo a la ventilación mecánica invasiva. Guía simple. CES Medicina, 34, 117-125. doi:http://dx.doi.org/10.21615/cesmedicina.34. COVID-19.16

Mosquera, F. E. (2020). SOPORTE RESPIRATORIO NO INVASIVO EN PACIENTES CON COVID-19i QUÉ DICE LA EVIDENCIA CIENTÍFICA? 


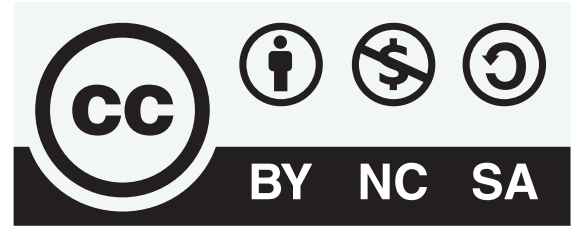

CREATIVE COMMONS RECONOCIMIENTO-NOCOMERCIAL-COMPARTIRIGUAL 4.0.

\section{CITAR ESTE ARTICULO:}

Pozo Rivadeneira, T. K., Matute Solís, M. F., Moreno Castro, F. I., \& Castillo Olvera, J. A. (2021). Oxigenoterapia a alto flujo en COVID-19. RECIMUNDO, 5(2), 37-45. https://doi.org/10.26820/recimundo/5.(2).abril.2021.37-45 